\title{
“ME DUERMO, PERO IGUAL APRENDO": EXPERIENCIAS EDUCATIVAS EXITOSAS DE JÓVENES SANTAFESINOS DE SECTORES BAJOS
}

\author{
“I fall asleep, but I learn": Successful educational experiences of low income \\ youngsters from Santa Fe, Argentina
}

http://dx.doi.org/10.22235/pe.v10i1.1358

\section{MARÍA N. SERRA ${ }^{1}$}

Universidad Nacional del Litoral (UNL), Santa Fe, Argentina

Recibido: 06-09- 2016

Correspondencia: María N. Serra, Universidad Nacional

Revisado: 29-09-2016

del Litoral (UNL). Argentina. Correo electrónico: mariaserra78@gmail.com

Aceptado: 25-11-2016

\begin{abstract}
Resumen: Este artículo busca identificar experiencias educativas exitosas de alumnos secundarios de la ciudad argentina de Santa Fe, que habitan en contextos desfavorables para la culminación de la educación obligatoria. Para ello, se realizaron entrevistas en dos escuelas locales a jóvenes de sectores socioeconómicos bajos y medios. Dicho trabajo de campo permitió reconocer, entre otras características, que los alumnos de sectores bajos perciben a la escuela como un lugar seguro, que los protege de la violencia del barrio y en el que adquieren habilidades y conocimientos específicos. También se observaron diferencias entre estos alumnos y sus pares de sectores medios, tanto en el modo en que establecen nexos entre la escuela y otros ámbitos de socialización, como en sus percepciones acerca del conocimiento, el trabajo y la universidad.
\end{abstract}

Palabras clave: secundaria, estudiantes de sectores bajos, contextos desfavorables, experiencias educativas, éxito

\begin{abstract}
This article explores successful educational experiences of high school students from the Argentinean city of Santa Fe, who live in unfavorable contexts to finish their compulsory education. In order to achieve this objective, interviews were conducted in two local schools, among low income students and middle-class students. This fieldwork allowed to recognize, among other characteristics, that the students of the low sectors perceive the school as a safe place that protects them from the violence of the neighborhood, and where they acquire specific skills and knowledge. Differences were also observed between these students and their middle-class peers, both in the way they establish links between school and other areas of socialization, as well as in their perceptions about knowledge, jobs and college.
\end{abstract}

Keywords: high school, low income students, disadvantaged situations, educational experiences, success

\footnotetext{
${ }^{1}$ Doctora en Ciencias Sociales por la Universidad Nacional de Entre Ríos, Argentina. Máster en Educación Intercultural por la Università Cattolica del Sacro Cuore, Milán, Italia. Docente de Metodología de la Investigación Social en la Universidad Nacional del Litoral (UNL) y en la Universidad Católica de Santa Fe (UCSF), Argentina. Becaria posdoctoral de CONICET. Sus principales trabajos de investigación se desarrollan con estrategias metodológicas cualitativas y se centran en problemas educacionales y migratorios.
} 


\section{INTRODUCCIÓN}

Las investigaciones sociológicas contemporáneas especializadas en el estudio de las trayectorias educativas - tanto las que analizan la educación considerada obligatoria en cada contexto nacional como las enfocadas en educación superior- se centran, por lo general, en analizar los determinantes sociales del fracaso o la interrupción de la experiencia escolar. Este trabajo busca centrarse en la situación inversa: experiencias escolares exitosas que transcurren bajo contextos desfavorables.

En términos generales, la culminación de la educación obligatoria está condicionada, entre otros elementos, por el clima educativo medio $o$ alto entre los adultos residentes en el hogar o a cargo del cuidado de los niños (Fernández Aguerre, 2009; Cervini, 2009); las expectativas paternas asociadas a la educación de los hijos (Pascual, 2008; Del Cueto, 2007); la residencia fija del grupo familiar y el trabajo estable del jefe de hogar (Dirección Nacional de Información y Evaluación de la Calidad Educativa, 2004). Por el contrario, como aspectos asociados al abandono y que aluden a atributos del niño, adolescente o joven, cuentan: ingreso al mercado laboral; maternidad o paternidad temprana (Anderson, 1993; Fernández Aguerre, 2009); exposición al riesgo de adicciones; habitar en entornos desfavorables (Román, 2009; Aristimuño, 2009), entre otros. En este sentido, la idea de este trabajo surge del interés por conocer cómo es la experiencia educativa de jóvenes que logran finalizar el ciclo obligatorio, a pesar de enfrentarse en forma cotidiana a las dificultades mencionadas.

Cabe remarcar que el éxito se asocia a la posibilidad de obtener el título secundario en un contexto desfavorable y no a los resultados obtenidos en evaluaciones institucionales. En tanto hecho social complejo, las trayectorias educativas de los alumnos de sectores socioeconómicos bajos que logran culminar no son necesariamente la versión opuesta de los recorridos que no llegan a ese fin. Y, por este motivo, se ha decido prestar atención a las experiencias exitosas.

La experiencia escolar, según Dubet y Martucelli (1998), se presenta como una prueba en la que los actores se ven obligados a combinar diferentes lógicas de acción en torno al espacio educativo. Aquí se buscará identificar aquellas lógicas que hacen que un grupo de alumnos que habita en contextos desfavorables termine los estudios. En otros términos, se busca indagar cómo se vinculan con y en la escuela, teniendo en cuenta, en particular, los nexos que establecen con otros ámbitos de socialización y las percepciones acerca de los conocimientos aprendidos, el mundo universitario y el mundo laboral.

\section{ESTRATEGIA METODOLÓGICA}

Esta investigación se centra en reconstruir la realidad tal y como la observan los actores (Hernández, Fernández y Baptista, 2003) dentro de su propio mundo social (Bertaux, 2005). Para lograr ese objetivo, a lo largo de los años 2013 y 2014, se entrevistaron 
alumnos de cuarto y quinto año de dos escuelas secundarias de la ciudad de Santa Fe. Ambos establecimientos son parroquiales y cuentan con un número de alumnos menor al de otras escuelas de la ciudad, pero se distinguen porque los alumnos que los frecuentan y los barrios en los que se ubican son socioeconómicamente diferentes.

Una de las escuelas se encuentra en Villa Centenario ${ }^{2}$ y la otra en Guadalupe. El primero es un barrio de sectores socioeconómicos bajos que se sitúa al sur de la ciudad, frente al margen del río Salado. Sus casas son precarias y no poseen todos los servicios. Algunas de sus calles no están trazadas y se inundan cuando las lluvias son abundantes. Como sucede en otros barrios de la ciudad con similares características, los eventos de violencia son frecuentes entre sus habitantes y repercuten en los medios de comunicación locales ${ }^{3}$. Por el contrario, Guadalupe es un barrio residencial de sectores medios y altos, no tiene carencias de infraestructura ni es epicentro de episodios de violencia.

Existen variables, como el nivel educativo familiar y el nivel económico de la familia, que influyen en las lógicas de acción bajo estudio. El coeficiente socioeconómico ${ }^{4}$ (CSE) agrega en un solo dato las variables antes mencionadas, al medir el nivel en el que se encuentran —en una escala del 0 al 100 - las familias de los alumnos que frecuentan los establecimientos estudiados. Al respecto, en el año 2006 la escuela de sector bajo registró un CSE de 76.16 (deficitario) ${ }^{5}$, mientras que la escuela de sector medio uno de 4.1 (bueno). Ambos coeficientes se corresponden con el hecho de que, entre otros indicadores relevantes, los alumnos del primer establecimiento reciben servicio alimentario y los del segundo no requieren de la escuela para poder cubrir esa necesidad básica en forma cotidiana.

Estos datos son coherentes con la información obtenida acerca del nivel educativo y de las actividades laborales que realizan los padres de los alumnos entrevistados. Los de sectores bajos se dividen entre quienes nunca fueron a la escuela y quienes no lograron terminar el primario o el secundario. Los de sectores medios, contrariamente, concluyeron en su totalidad la escuela secundaria y, en algunos casos, realizaron estudios terciarios o universitarios. A su vez, la diferencia de niveles educativos se refleja en los trabajos que realizan los padres de uno y otro grupo, ya que en el primero

\footnotetext{
${ }^{2}$ Este artículo se escribió a partir de algunos de los resultados del trabajo de campo desarrollado en Villa Centenario para el proyecto de investigación "El ejercicio de ciudadanía en contextos de vulnerabilidad social". Este proyecto, que fue financiado por la Universidad Católica de Santa Fe (UCSF), se realizó bajo la dirección del Mg. Eduardo Nicolás Kinen, con la participación de la autora del presente artículo como investigadora y con la colaboración de las alumnas Mercedes Banchio y Florencia Ordóñez.

3 "Alumnos y maestras en el suelo para protegerse de un tiroteo" (El Litoral, 16/04/2014). "Escuela jaqueada por ladrones" (El Litoral, 13/12/2014). "Homicidio en la Villa Centenario" (El Litoral, 25/10/2014). "Los cronos imponen su ley en la Villa del Centenario" (El Litoral, 24/4/2014).

4 Índice elaborado por el Sistema Informático Integral para el Planeamiento y la Administración de Recursos (iSIEME) del Ministerio de Educación de la Provincia de Santa Fe.

${ }^{5}$ Este último dato corresponde a la escuela primaria. Se utiliza como válido en este trabajo en tanto los alumnos del secundario son, en su mayoría, ex alumnos del primario del mismo establecimiento educativo.
} 
predominan actividades que se caracterizan por una mayor informalidad (pescadores, albañiles y empleadas domésticas) y, en el otro, por una mayor formalidad (empleados públicos y de comercios, profesionales autónomos, maestras y docentes).

La muestra, en tanto selección basada en criterios (LeCompte y Preissle citado en Maxwell, 1996, p. 68), se conformó en función de dos requisitos. El primero fue la selección de los alumnos con base en su pertenencia a dos contextos socioeconómicos opuestos, requisito que hizo posible hacer comparaciones entre los dos grupos, así como comprender la particularidad de la experiencia educativa de los alumnos de sectores bajos. El segundo requisito fue el que los alumnos estuvieran frecuentando los últimos dos años de la escuela secundaria (cuarto y quinto año), ya que quienes llegan a esa instancia tienen menos probabilidades de abandonarla que quienes están en los primeros años.

La muestra estuvo compuesta por once alumnos de cuarto año (siete mujeres y cuatro hombres de entre 16 y 20 años de edad) y cinco alumnos de quinto año (todos hombres de entre 17 y 19 años) de la escuela de sector socioeconómico bajo. Y, de la escuela de sector medio, la selección incluyó a once alumnos de cuarto año (cinco hombres y seis mujeres de 16 años) y tres mujeres de 17 años que se encontraban cursando quinto. Por su parte, se seleccionaron observadores claves: el preceptor de la escuela y una psicopedagoga en el primer centro, y uno de los preceptores de los alumnos en el segundo.

Al centrarse en dos escuelas parroquiales ${ }^{6}$, la investigación dejó fuera aquellas públicas y laicas. Así, se excluye la perspectiva que poseen, tanto de la escuela como de su tránsito por ella, los alumnos de esos espacios, que se caracterizan —a diferencia del parroquial- por no estar vinculados a la dimensión religiosa (misas, horas de catequesis, clases de religión, retiros espirituales). Por otra parte, en tanto se trata de un estudio cualitativo, no se pretende generalizar los resultados a todos los jóvenes estudiantes secundarios de la ciudad de Santa Fe.

Las técnicas utilizadas para la recolección de información, en lo que refiere a los alumnos, fueron la entrevista grupal y una breve encuesta individual. Se seleccionaron grupos de tres a cuatro personas que compartieran el mismo curso, con la idea no solo de recabar una mayor cantidad de información en menor tiempo, sino también por la posibilidad de motivar el diálogo entre ellos y la aparición de formas compartidas de interpretar los ejes de la indagación. En lo que refiere a los observadores claves, se hicieron entrevistas en profundidad de manera individual, ya que se consideró que cada uno de ellos podía dar una visión específica y externa acerca de los temas de interés establecidos.

\footnotetext{
${ }^{6} \mathrm{El}$ hecho de que sean de gestión privada no implica que los alumnos tengan la obligación de pagar una cuota. El Estado provincial, de acuerdo a lo que se establece en el artículo 16 de la Ley $n .{ }^{\circ} 6427$, se hace cargo del presupuesto de aquellas escuelas parroquiales frecuentadas por alumnos socioeconómicamente desfavorecidos.
} 


\section{ALGUNOS CONCEPTOS CLAVES}

La experiencia escolar, en tanto concepto clave de este trabajo, se concibe como el modo en el que se articulan y combinan diferentes lógicas de acción en torno al espacio educativo (Dubet y Martucelli,1998). Dichas lógicas, según los citados autores, pueden ser de estrategia, socialización y subjetivación. La primera está ligada a lo que Weber denominó acción racional con respecto a fines; la segunda, por su parte, a la interiorización de normas a través del desempeño de roles establecidos; y la última, al proceso - relativamente independiente de las funciones y normas objetivas-que está asociado al cuerpo, a la maduración biológica y al desarrollo psíquico. En este trabajo se tendrá en cuenta, principalmente, la segunda dimensión.

Como ha sido documentado en investigaciones especializadas en el proceso de transición a la vida adulta (Elder,1978; Hareven, 2000; Mayer, 2004), la infancia y la adolescencia son etapas del curso de vida relativamente estandarizadas; es decir, que los niños transcurren sus días en instituciones que homogeneizan sus comportamientos. Sin embargo, en el contexto de las sociedades contemporáneas signadas por el declive institucional (Dubet, 2007), la socialización de los niños y adolescentes se desarrolla en otras esferas más allá de las institucionales.

Si bien la escuela sigue ocupando un lugar central como reguladora de la infancia y adolescencia, quienes por allí transitan están inmersos en procesos de socializaciones múltiples que incluyen, además, a la familia y al grupo de pares (Lahire, 2007). El grupo de pares, en particular, representa la oportunidad de ejercer nuevos roles: el de amigo, compañero de equipo, de murga o taller (Salvia, 2009). Ahora bien, en contextos desfavorables para la culminación de la educación obligatoria, como el aquí estudiado, los nuevos roles aprendidos "entre amigos" pueden devenir en ingresos tempranos al mundo de la delincuencia y de las adicciones (Observatorio Argentino de Drogas, 2010) y constituirse, entre otros, en motivos de abandono escolar.

El sector socioeconómico de pertenencia condiciona las posibilidades y recursos que se ponen en juego a la hora de vincularse con otros. La socialización entre pares muchas veces se produce en espacios accesibles solo para pocos. Los jóvenes de sectores medios, por ejemplo, realizan actividades deportivas, lúdicas o artísticas que sus coetáneos de sectores bajos tienen vedadas. Así, mientras los primeros entablan lazos y tienen experiencias enriquecedoras en términos de capital social y cultural, los segundos transcurren la mayor parte de su tiempo sin contactos significativos con espacios sociales diferentes al suyo, lo que reproduce la fragmentación laboral, educativa y residencial en la que están inmersos.

La noción de fragmentación educativa aquí considerada, en particular, reconoce que cada espacio educativo se presenta como un fragmento. Más allá de la clásica distinción por sectores sociales, un fragmento se define, según Tiramonti (2005), como: 
Un espacio auto referido, organizado alrededor de creencias, valores particulares que construyen un específico patrón de socialización para los chicos y donde no aparecen diálogos, interlocuciones a un elemento central común.... No existen referentes comunes. Y, por sobre todo, la idea es que lo que pasa dentro de cada uno de los fragmentos es incomparable con lo que pasa en el otro fragmento (p.99).

De esta forma, cada espacio educativo, más allá de compartir un determinado sector social, es un fragmento a analizar en forma separada para poder entender los recorridos concretos de los alumnos dentro de ese marco. Pues, como señala esta autora, la fragmentación tiene mucho que ver con la asociación establecida entre la escuela y la comunidad sociocultural, es decir, entre la escuela y el tipo de población que está atendiendo (Tiramonti, 2005, p. 100).

La educación, en paralelo a su fragmentación, sigue siendo un mecanismo eficiente de reproducción de la pobreza y desigualdad de origen (Bourdieu y Passeron, 2003). Los padres pobres envían a sus hijos a escuelas para pobres y, por lo mismo, las trayectorias laborales y de vida de estos jóvenes son empobrecedoras (Salvia, 2009). Sin embargo, a través de una lectura microsocial como la que se realizará aquí, se podrá observar que la distribución de oportunidades y la acumulación de desventajas (Saraví, 2004) no se da de forma homogénea entre los jóvenes de sectores bajos. Estos, en muchos casos -incluso compartiendo un mismo origen social, experiencias y perspectivas a futuro-, se distinguen entre sí por frecuentar o no la escuela y por tener trayectorias laborales y de vida más o menos exitosas dentro de su propio marco.

En lo que refiere a las trayectorias educativas, Terigi (2015) las define como reales y teóricas. Las primeras se asocian a los recorridos concretos que realizan por la escuela los actores estudiados, mientras que las segundas se vinculan al diseño del sistema escolar y a sus expectativas en torno a los años de estudio a transitar, a la edad del alumno al momento del ingreso y del egreso, y a los aprendizajes previstos por el currículum. En este marco, las experiencias educativas exitosas que se estudian son aquellas que, a pesar de desarrollarse en contextos desfavorables para la culminación de la educación obligatoria, logran hacer confluir las trayectorias educativas reales con las teóricas, las que, de acuerdo a lo que se pudo observar, se adaptan al contexto desfavorable en el que se encuentran.

Los estudios sobre cursos de vida y transiciones educativas permiten reconocer los límites y las oportunidades que producen las instituciones -entre ellas la escuela secundaria - por las que transcurren los alumnos (Fernández Aguerre, 2010). Es decir que, como se identificará en las experiencias de los alumnos, tanto en la trayectoria del secundario como en el pasaje a la universidad, los estudiantes y sus familias toman una serie de decisiones y elecciones que están condicionadas por las opciones y 
posibilidades con las que cuentan de acuerdo al contexto socioeconómico en el que están inmersos.

Es por ello que el grupo de alumnos de sectores socioeconómicos bajos que aquí se estudia requiere ser observado y analizado de acuerdo a las desigualdades persistentes que vivencia (Tilly, 1998), no solo en comparación con los jóvenes de sectores medios, sino también entre sus propios pares (estén o no escolarizados). Si bien se asume como un hecho que los jóvenes más pobres no pueden construir biografías de éxito similares a las de los jóvenes de sectores medios o altos, se reconocen diferentes modelos de éxito (Lahire, 2010) al interior de los grupos menos favorecidos: los que terminan la escuela pese a las condiciones adversas, los que continúan estudiando una vez concluida la escuela secundaria o los que logran una inserción laboral estable y, por ende, ingresos fijos.

Más allá de las características estructurales mencionadas, la institución educativa se encuentra en un momento de cambio. Los problemas, conflictos y violencias emergentes en contextos desfavorables para concluir la educación obligatoria repercuten en los establecimientos escolares, y han cobrado primacía en la agenda escolar, pública y de los medios de comunicación por sobre la discusión del conocimiento (Mayer, 2009). En el escenario de masificación de la escuela y de las dificultades en torno a la definición de su rol social como transmisora de cultura, Tenti (2000) sostiene:

La vieja escuela media reservada a las elites hoy debe responder a la demanda de nuevos contingentes de ingresantes.... Los viejos dispositivos que regulaban la relación profesor-alumno y la relación con el conocimiento, garantizaban la autoridad pedagógica y producían un orden institucional, se erosionan cuando no saltan por los aires y dejan de ser eficientes y significativas en la vida de los actores implicados (p.1).

Bajo este escenario, se experimenta un momento incierto en lo que respecta al lugar social que ocupa la escuela y a lo que se puede esperar de dicho espacio institucional. Al respecto, Carusso y Dussel (1996) sostienen: "la identidad que producía la escuela existía y luchaba contra otras para imponerse... pero ahora la primacía de lo escolar en la definición de las identidades sociales se ha roto" (p.47). En otras palabras, la discusión acerca de la escuela como transmisora de conocimientos muchas veces queda relegada a los nexos que se establecen entre los diferentes actores en juego, y a las tensiones que se producen entre el interior de la institución y el exterior (barrio, familia, redes sociales) en el proceso de constitución del joven como alumno.

Habitar la escuela hoy se percibe, desde el punto de vista de niños y adolescentes, como una experiencia de carácter obligatorio en la que se pueden hacer buenos amigos, pero son pocas las referencias al proceso de adquisición de saberes útiles para la construcción de biografías menos riesgosas. Los mecanismos de integración social 
(educación, trabajo), que permitieron la construcción de biografías estables en contextos de primera modernidad, parecieran tambalearse sin que emerjan canales sustitutos. Igualmente, más allá del escenario que atraviesa la escuela en general, en este trabajo se evidencian algunos de los matices que dicho proceso de crisis y transformación institucional en fragmentos puede presentar en el tránsito por la escuela de jóvenes de sectores socioeconómicos bajos.

\section{LÓGICAS DE ACCIÓN EN ALUMNOS DE SECTORES BAJOS}

Estas páginas se centran en indagar las lógicas de acción que desarrolla en torno a la escuela un grupo de jóvenes de sectores bajos que no la abandonan. Se tienen en cuenta, específicamente, los nexos establecidos entre la escuela y otros marcos de socialización como el barrio, el grupo de pares y la familia, así como las percepciones acerca de los conocimientos adquiridos, el mundo universitario y el laboral.

\section{En el barrio}

De acuerdo a lo que se pudo identificar, los alumnos de sectores socioeconómicos bajos (de ahora en más ASB) viven en los alrededores de la escuela y asumen que el barrio es el mismo contexto en el que transcurre su vida cotidiana y estudiantil. Sin embargo, ellos distinguen lo que sucede en las calles del barrio de lo que experimentan en la escuela. Esta se percibe como un lugar seguro en el que se puede hacer amigos, mientras que por fuera hay personas peligrosas y situaciones de riesgo.

E (entrevistador): ¿Cuesta hacerse amigos en el barrio?

A1 (Alumno 1): Sí.

A2: Es que no hay tantas chicas de la edad de nosotros, sino que más grandes y son muy peligrosas.

E: ¿Peligrosas?

A2: Sí. Peligrosos, porque hay más hombres que mujeres.

E: ¿Qué sería peligroso?

A2: Claro, que andan a los tiros, todo eso. (ASB, $4^{\circ}$ año)

Los ASB entrevistados prefieren los lazos establecidos al interior de la escuela y consideran que el afuera es un lugar a evitar. Habitar en un contexto barrial como el aquí estudiado, en el que la violencia está naturalizada como mecanismo de resolución de 
conflictos y como canal (delitos) para conseguir cosas socialmente valiosas, les permite reconocer las diferentes lógicas de acción que se manejan al interior de los espacios sociales por los que transitan.

$\mathrm{E}:$ ¿Te gusta juntarte con amigos?

A: Sí, me junto. Pero no me dejan salir mucho, por el tema de cómo está el barrio.

$E: A h$.

A: Estoy más adentro que afuera porque afuera no se puede andar.

$\mathrm{E}: \mathrm{Y}$ tus amigos en general, ¿de dónde son?

A: Son de la cuadra nomás.

E: ¿Son los de la escuela?

A: Sí, son los de la escuela. (ASB, 5ำ año)

Los alumnos de sectores medios (de ahora en más ASM) viven en promedio a 16 cuadras de la escuela, por lo cual, a diferencia de los ASB, no visualizan un nexo estrecho entre su lugar de residencia y el de estudio. Los ASM se desplazan por diferentes barrios (para ir a la escuela y para realizar otras actividades diarias) y por ello tienen la percepción de que las situaciones de inseguridad experimentadas se localizan en diferentes puntos de la ciudad, en sus propias palabras, en "la calle", en "todos lados". Asimismo, para ellos, las personas que cometen delitos son desconocidas, a diferencia del registro que tienen los ASB, quienes conocen y caracterizan a dichos sujetos.

E: ¿Fundamentalmente dónde les han robado?

A1: En la calle.

E: ¿Cuando van y vienen de la escuela o en otro ámbito?

A1: En todos lados.

$\mathrm{E}:$ ¿Les ha pasado alguna experiencia personal?

A2: Sí, a mí sí. 
A2: A mí me apuntaron con un arma y me quisieron robar.

A3: A mí también me quisieron robar.

A3: O ves casos. Vas caminando y ves que pasan dos en moto y le roban a alguien y eso también te agarra como una pequeña inseguridad. (ASM, $4^{\circ}$ año)

En síntesis, los ASB perciben que los peligros se encuentran en el propio barrio y que la escuela les permite evitar el peligro externo. La identificación de lógicas de acción (seguridad/riesgo) producidas en la escuela y en las calles del barrio se vuelve comprensible no solo porque el barrio es el mismo contexto en el que transcurre su vida cotidiana y estudiantil, sino también por la cercanía y familiaridad con los actores reconocidos como "peligrosos".

\section{Con los pares}

Los dos grupos de alumnos dijeron que les gusta frecuentar la escuela, en tanto es un espacio en el que se "está con amigos". Sin embargo, los ASM hicieron hincapié en el hecho de conocer a sus compañeros y amigos desde chicos, lo que permite inferir una historia compartida como grupo. Por el contrario, los ASB evidenciaron la imposibilidad de un recorrido similar debido a los abandonos y repeticiones.

A1: Pasa que nos conocemos todos muy de chicos, venimos todos desde el materno y tenemos un vínculo...

E2: ¿Vos empezaste en el secundario o hiciste...?

A2: No, empecé en $4^{\circ}$ grado.

E1: Así que hace rato que todos vienen acá.

A2: Sí, nosotros venimos del materno, ya de los tres años, y él arrancó en $4^{\circ}$ o $5^{\circ}$, por ahí. (ASM, 4ํaño) 
E1: ¿Qué pasó con los que abandonaron? Porque es notable, como vos dijiste, empezaron $40^{7} \ldots$

A2: Algunos repitieron, y todavía siguen viniendo.

A3: Ponele que son cinco, pero los demás dejaron.

A2: La mayoría fueron padre y madre. Franco...

A3: No, pero Franco dejó la escuela porque repitió y no quiso venir más.

E2: Y después, ¿qué hacen?

A2: Nada, trabajan. Albañiles es lo que hacen.

A3: Lo único. Lo más accesible, lo más fácil de encontrar.

E1: ¿Se dedican a otras cosas?

A3: Franco hacía panadería.

E2: ¿Acá en el barrio también?

A3: Sí, porque acá hay una panadería. (ASB, 5aano)

Los ASB, a diferencia de los ASM, no tienen una historia compartida. En su propia experiencia educativa, el número de alumnos se va reduciendo con el paso de los años, ya sea por la llegada de un hijo, el inicio de actividades laborales informales, las repeticiones de año o bien por las constantes migraciones de un barrio a otro. Al respecto, dos de las alumnas entrevistadas evidenciaron que la migración interbarrial se presenta con relativa frecuencia, en algunos casos, luego de vivenciar conflictos interpersonales.

E1: ¿Vos vivís en Yapeyú ${ }^{8} ?$

A1: Sí, hace poquito.

\footnotetext{
${ }^{7}$ En el momento de la entrevista eran cinco los que habían llegado a 5ํaño.

${ }^{8}$ Barrio del sector socioeconómico bajo lindante con Villa Centenario.
} 
E2: Ah, vos vivías acá antes.

A1: Sí, en Varadero ${ }^{9}$, en el barrio aquel.

E1: Acá cerca... ¿Vos también venís de Varadero?

A2: Sí.

E2: Y los amigos que tienen, además de la escuela, ¿son parientes y amigos del barrio?

A2: De los dos. De la escuela y del barrio.

E2: Y vos, ¿amigos de Yapeyú tenés?

A1: No, si hace una semana que estoy allá... (ASB, 4ํaño)

E1: ¿Dónde viven tus amigas?

A2: En barrio San José, allá por Las Flores ${ }^{10}$.

E1: Ah, o sea que te juntás los fines de semana.

A2: Sí, voy a verla.

E1: ¿Y dónde la conociste?

A2: No, es del barrio. Hace muchos años yo soy amiga de ella.

E1: Vivía acá [Varadero y Villa Centenario están divididos por una calle], vivía en Varadero antes, y ella después se mudó.

A2: Hace poco se fue.

E1: Ah.

\footnotetext{
${ }^{9}$ Barrio del sector socioeconómico bajo ubicado al noroeste de la ciudad de Santa Fe.

10 Barrio del sector popular ubicado al norte de la ciudad de Santa Fe.
} 
A2: Por problemas

E1: ¿Por qué se tuvo que ir?

A2: Problemas más que nada, por los hermanos, todo eso. Había mucho quilombo y se tuvieron que ir. (ASB, $4^{\circ}$ )

El grupo de pares no se limita a los compañeros de escuela, sino que también incluye a aquellos jóvenes que se consideran conocidos. Entre los ASB, los conocidos son personas que han muerto por conflictos entre bandas, así como vendedores de drogas, las cuales, a su vez, son cercanas a su cotidianeidad (vecinos o parientes).

En ambos casos, son jóvenes que no solo no han terminado la escuela, sino que también son aquellos con los que se quiere establecer un cierto distanciamiento.

$\mathrm{E}:$ ¿Conocen gente herida?

A1: Por mi parte ya mataron a dos o tres de mis parientes con esos problemas.

E: ¿Qué eran tuyos?

A1: Primos.

E: ¿Formaban parte de alguna banda, o fue una bala perdida?

A1: No, de bandas.

E: ¿Qué edad tenían?

A1: 18,20 y 22 .

E: ¿Vos tenías relación con ellos, o...?

A1: Sí.

$\mathrm{E}:$ ¿Los querías?

A1: Y sí. ĺbamos a la misma escuela y todo. (ASB, 4ํaño)

$\star *$

A1: Primero, el que vende no terminó la escuela, por algo está vendiendo. 
E: Ustedes los identifican, ¿saben quiénes son?

A1, A2 y A3: Sí.

A2: Sí, la policía sabe quiénes son también, porque hay una situación de tiroteo y ya le saben el nombre, apellido y todo.

E: ¿Cómo ve el barrio a estos tipos que trafican droga?

A1: Es la molestia que da el vago, porque si vos ves que el vago vende o se droga y no molesta a nadie... no me importa, pero acá molestan. Ese es el tema.

A2: Ponele, cuando acá hay situación de tiro, no van a ir frescos, ya vienen re drogados a tirar. (ASB, 5ำ año)

Los ASM, por su parte, conciben que los conocidos son aquellas personas con las que, si bien no existe un nexo de confianza, se interactúa como miembros de una misma escuela o como participantes de otro espacio de socialización, como el deporte. En ningún momento los conocidos aparecen como personas que han abandonado la escuela ni como protagonistas de hechos delictivos, sino como aquellos con los que se tiene un nivel de confianza menor, en tanto no se conocen elementos biográficos que sí identifican en sus propios amigos.

A: Por ejemplo, yo juego en Lasalle ${ }^{11}$ y ahí juegan todos los que van a la escuela. Empecé este año a jugar ahí, mi hermano ya juega ahí. Empecé a tener conocidos, pero era un grupo muy cerrado.

E: ¿Qué jugas?

A: Fútbol. Es medio cerrado. Entonces yo le decía a mi hermano, ¿para qué me cambié? Y él me decía "es que no te conocen y ellos se conocen", como nosotros, así. Entonces es difícil aceptar a uno de afuera.

E: ¿A qué le atribuís esa dificultad? ¿Por qué creés que es difícil?

\footnotetext{
11 Escuela parroquial y espacio deportivo de la ciudad de Santa Fe a los que frecuentan alumnos primarios y secundarios de sectores medios y altos.
} 
A: Mirá, acá a Juan lo conozco, se dónde vive, de qué trabajan los padres. Igual que a Bruno. Y ellos a mí. Entonces si viene un chico nuevo al grupo, yo no sé dónde vive, no conozco nada de él, entonces ahí es cuando entra desconfianza.

E: ¿Acá también son cerrados los grupos?

A: En general acá también. (ASM, 4ํaño)

En síntesis, para los ASB los pares son tanto sus compañeros y amigos de la escuela como aquellos conocidos que la han abandonado por diferentes motivos (migraciones barriales, paternidad o maternidad temprana, ingreso al mundo de las drogas). Una diferencia a destacar entre los dos grupos de alumnos es el hecho de que los ASM registran una historia compartida desde los primeros años de la escuela, mientras que los ASB no tienen esa experiencia de largo plazo, porque incluyen dentro de la propia trayectoria escolar la disminución numérica que fue sufriendo el grupo a raíz de las repeticiones o los abandonos.

\section{Con la familia}

Los ASM no registraron explícitamente ${ }^{12}$ a la familia como un agente que interviene en lo que sucede en la propia experiencia educativa. En cambio, los ASB sí perciben la insistencia de los padres o mayores a cargo para que terminen la escuela. Además, en relación a algunos miembros de la propia familia que no estudiaron, observan que carecen de ciertas habilidades con las que ellos cuentan gracias a la escuela.

E: En el caso de las dos familias de ustedes, ¿les insisten para que terminen la escuela?

A: Sí.

A1: Mi papá sí. Mi mamá también, porque mi mamá no terminó la escuela y mi papá sí.

A2: Sí, mi mamá también. Quieren que terminemos para que por lo menos tengamos algún futuro.

\footnotetext{
12 En tanto en este sector social está naturalizada la frecuencia y la culminación de la escuela, no tematizan prácticas y eventos que dan por hechos.
} 
A1: Mi papá me dice que la tengo que terminar, me insiste para que la termine. Si necesito un trabajo o algo, mostrar que yo terminé la escuela. Como mi mamá no la pudo terminar, entonces ella también me insiste. Porque mi mamá no sabe leer, no sabe escribir.

E: ¿Vos la ayudás a tu mamá con eso?

A1: Sí, todas la ayudamos a mi mamá.

E: ¿Vos notás entonces que el hecho de terminar la escuela o concurrir a una escuela te da ciertas herramientas para manejarte?

A1: Sí.

E: ¿Por ejemplo?

A1: Yo puedo darme cuenta de un montón de cosas que mi mamá no se puede dar. Porque ella va a comprar algo y tiene que hacer una cuenta $o$ algo y ella no sabe. Ya una vez fue al súper y tuvo que pagar ella y, al no saber, le sacaron plata a ella. Ahí en el súper. Entonces después se sentía mal por no saber eso. Hay muchos de mi familia que no terminaron la escuela, aparte de mi mamá. (ASB, 4ํaño)

A: $Y$ vos lo ves a mi primo, lo veo a mi primo ahora $y$ anda con una 9 [milímetros, en referencia a un arma] en la cintura, en la calle y nada que ver a como era antes. Yo me alejé de todos ellos allá cuando vivía, me vine para acá y mi tío me puso a estudiar [...] vos lo ves ahora con una 9 y anda a los tiros... (ASB, 5ำ año)

La psicopedagoga de los ASB también percibe una relación entre el interés de los padres por la continuidad de los hijos en la escuela y los resultados obtenidos. Asume que, en algunos casos, el abandono escolar está asociado a una valoración de la escuela como no obligatoria e innecesaria.

Psicopedagoga ASB: A nivel del contexto, digamos que... creo que tenemos una dificultad seria, que es que los papás de los chicos no reconocen que la escuela secundaria es obligatoria y no la perciben como 
necesaria. Entonces, ante distintos obstáculos que se presentan, ya sea familiares o educativos... suele decir "bueno, si es para problemas yo no lo llevo y ya está". Si tienen algún problema, ya sea familiar o dentro del barrio... ellos quizás se trasladan y de la escuela se olvidan. Hay que ver dónde está [viviendo en qué barrio] con la asistente social y ver cómo solucionar esto.

Los ASB no solo registran el interés de algunos miembros de la familia por la propia continuidad educativa, sino que también identifican las limitaciones que poseen por no haber ido a la escuela. Esta situación tiene un efecto aleccionador en relación al propio futuro: "si termino la escuela evito experiencias indeseadas como no saber contar dinero o andar con un arma en la cintura".

En síntesis, los ASB registran el interés familiar por la continuidad educativa y, al mismo tiempo, reconocen las habilidades y conocimientos que ellos han desarrollado gracias a la escuela. Las dificultades familiares en torno a la ausencia de escolarización no las mencionan como un obstáculo, sino que, por el contrario, aparecen como una lección acerca de los pasos a evitar para no repetir experiencias indeseadas.

\section{De acuerdo a los aprendizajes adquiridos}

Los ASB consideran que en la escuela se aprende. Más allá de asumir que la mayor parte del tiempo se aburren y duermen, observan e identifican diferentes habilidades y conocimientos adquiridos. Saben escribir, hacer cuentas y hablan mejor que quienes no van o no han ido a la escuela. Además, consideran que conocen más palabras, lo cual les permite interactuar y adaptarse a diferentes situaciones y tipos de personas (médicos, profesores) en donde se requiere una mayor competencia lingüística.

A1: Me gusta por ahí venir a la escuela, porque me aburro en mi casa.

E: ¿Y qué tenés acá en la escuela que te divierte?

A2: Me hablo con todos más que nada.

A1: Aparte podés aprender.

E: ¿Aparte qué?

A1: Aprendés.

A2: Claro... Por ahí me duermo, pero igual aprendo. (ASB, $4^{\circ}$ año) 
A1: Yo tengo más posibilidades de conseguir un trabajo mejor.

A2: O ella entiende mejor las cosas académicas. Nosotras hablamos bien y las otras hablan como negras, digamos. O van a un médico, ponele, y les explica así, así, y ellas no entienden, porque no saben las palabras, todo eso.

A1: No entienden las cosas que les explican. Yo al saber ya más cosas puedo llegar a entender un poco más que ellas. (ASB, 4ํaño)

E: Y... ¿vos ves la diferencia? ¿En el modo de hablar? ¿En qué más?

A1: Cuando escribe, jaja.

E: O sea que te permite escribir bien...

A2: Adaptarte en distintos ambientes.

E: ¿Qué sería eso?

A2: Yo capaz que con ellos no hablo así, como estoy hablando ahora, hablo "eeh...", así, boludeando ${ }^{13} \ldots$ (ASB, 5ำ año)

Los ASM, por el contrario, asumen que la escuela solo sirve para hacer amigos y para la obtención del título (para poder seguir estudiando o conseguir un trabajo). Idea que también sostiene el preceptor cuando hace alusión a la inutilidad de la escuela —en términos de aprendizaje - para los alumnos del sector social al que estos pertenecen. Para este grupo, el hecho de frecuentar la escuela solo tiene un sentido burocrático, en tanto esta no va más allá de los saberes que ya tienen garantizados por su sector de origen.

${ }^{13}$ Boludo es una palabra muy usada en Argentina, se podría considerar un sinónimo de tonto. 
E: ¿Para qué piensan que les sirve venir a la escuela?

A1: Para tener un título.

A2: Sí, porque hoy en día si vos no tenés la secundaria terminada vos no podés ni estudiar ni trabajar.

A3: Tener un trabajo decente.

A1: Ni basurero podés ser si no terminás.

E: Aparte de eso ¿alguna otra cosa que sientan que la escuela les da o les otorga?

A1: No.

A2: El grupo de amigos... (ASM, 4ํaño)

**

Preceptor ASM: en una época era para que tengan más conocimientos a nivel general. Ahora, yo lo veo como un paso que mucho mucho no sirve, que es un paso de la primaria a la universidad.

E. ¿Por qué decís "mucho mucho no sirve"?

Preceptor ASM: Porque los contenidos son pocos, son bajos.

En síntesis, en lo que refiere al aprendizaje en la escuela, los ASB consideran que aprenden y que, gracias a la escuela, pueden adquirir conocimientos (nuevas palabras y capacidad de hacer cuentas) y habilidades (de escritura y comunicativas) que sus pares o familiares que no han concluido el secundario no poseen. Los ASM, contrariamente, no valoran a la escuela como un espacio de aprendizaje, sino como una instancia que los habilita, en términos burocráticos, para la búsqueda laboral o para el ingreso universitario.

\section{Con el mundo universitario}

En lo que refiere a la relación entre la escuela secundaria y la universidad, alumnos de ambos centros manifiestan querer seguir estudiando una vez finalizado el ciclo en curso. Sin embargo, solo los ASM reconocen que la escuela no les otorga el nivel necesario 
para tener una transición a la universidad sin dificultades, mientras que los ASB no tematizan como problema el hecho de poseer o no las competencias que se requieren para frecuentar la universidad.

A1: [En comparación] con otras escuelas yo me siento como que a veces nos faltan cosas. Por ejemplo, ahora con los ingresos [universitarios] hay escuelas que están muchísimo más avanzadas que nosotros en algunas materias, entonces vos te das cuenta que ellos saben más y no les cuesta tanto. (ASM, 5ำ año)

Al respecto, la psicopedagoga de los ASB y el preceptor de los ASM hacen hincapié en lo que ellos llaman "los contenidos", lo "pedagógico" o la "exigencia". En uno y otro caso, asumen que lo que aprenden los alumnos en la escuela no es suficiente para habilitarlos a estudios terciarios o universitarios. La primera explicita que un número ínfimo de sus egresados se encuentra en dicha situación; el segundo incluso considera la "inutilidad" actual de la escuela secundaria en ese aspecto.

Preceptor ASM: Yo veo, por ejemplo, los chicos que [deciden estudiar] Medicina... Todos se van a una academia a preparar. Los chicos que van a ingresar a una Facultad de Ingeniería o algo de eso, se van a preparar en matemática, en física, en química. Entonces están todos preparándose aparte de la escuela... La escuela tendría que ser la que les sirve para dar ese paso a la universidad.

Psicopedagoga ASB: Veo que todavía nos falta mucho. Es decir, para tener competencias para la continuidad en un instituto terciario. Hoy en día tenemos chicos en el instituto de educación física, creo que dos, si mal no recuerdo... hay uno de los chicos (se refiere a los egresados de la escuela) que está estudiando Medicina. Esperemos que les vaya bien, pero creemos que falta mucho desde lo pedagógico.

Los alumnos no egresan con las competencias necesarias para seguir una carrera universitaria. Sin embargo, los ASM reconocen esta situación y buscan soluciones individuales (frecuentan academias particulares), mientras que los ASB no lo asumen como problema en tanto el eje está puesto en terminar y no tanto en qué condiciones hacerlo. Como evidencian las experiencias aquí relatadas, la desarticulación que existe entre ambas instituciones educativas solo puede ser "reanudada"-como lo demuestran estos alumnos- a través de acciones individuales. Y, dado que los ASB dependen de 
las oportunidades y opciones que les brinda el sistema educativo, quedan excluidos de esta lógica de enmienda.

Los actores involucrados en la educación de los ASB, a diferencia de estos, sí observan una desarticulación entre la escuela secundaria y la universidad. Sin embargo, a la hora de establecer sus causas, emergen dos lógicas opuestas: una estructural y otra individual. La primera, cuando asumen que el problema lo tiene la institución (la escuela no otorga los saberes necesarios para ingresar a la universidad) o bien cuando consideran que el sector social condiciona el horizonte de posibilidades (solo los del Centro pueden seguir carreras como Abogacía o Medicina). La segunda, en cambio, cuando depositan el problema en los alumnos: no tienen hábitos de estudio o eligen carreras que conocen o perciben cercanas (estudiar para ser maestra o policía, por ejemplo).

Preceptor ASB: Por ahí, sabemos la carga que es una carrera como Medicina, las cargas horarias de estudio que tienen que tener [...] No tienen el hábito de estar sentados media hora leyendo la carpeta, entonces imagínate meterse en semejante carrera. Está la mujer que te dice que va a ser maestra, son también las carreras que ellos conocen. Creo que las ven más accesibles que Abogacía, Medicina, Ciencias Económicas. Ni te las nombran como posible opción. Las ven lejanas.

E: Y para ellos ¿quién hace esas carreras?

Preceptor ASB: Ah, buena pregunta. Y no te sabría decir, pero yo calculo que eso lo hacen los que tienen plata, digamos. Eso lo hace gente del Centro. Hay ese preconcepto.

En síntesis, hay una desarticulación entre la secundaria y la universidad. Los actores de ambas instituciones reconocen que los alumnos no egresan con las competencias necesarias para poder seguir estudiando. Frente a esta situación, los ASM logran enmendar dicha falta frecuentando academias particulares, mientras que los ASB no lo plantean como posibilidad, en tanto dependen de las opciones y oportunidades que les otorga la institución en la que se encuentran. En lo que refiere a las causas de dicha desarticulación, los actores fluctúan entre el reconocimiento de factores estructurales que condicionan las prácticas observadas y la culpabilización de los alumnos por no tener ciertas competencias o habilidades, como el hábito de estudio. 


\section{Con el mundo laboral}

Los ASB consideran que el título secundario no les garantiza trabajar en algo que les guste, pero sí que les da más posibilidades, no solo de conseguir un trabajo con un sueldo fijo, sino también de realizar actividades más gratificantes y menos sacrificadas que las que llevan a cabo quienes no han terminado los estudios.

E: [Las personas que conocen que han terminado la escuela] ¿ustedes ven que han podido conseguir un trabajo?

A1: Sí, aparte tienen un sueldo fijo.

A2: Sí, aparte pasa más por las ganas que le mete cada uno. Si vos querés trabajar, vas a hacer lo posible por trabajar.

A1: Vos capaz terminando la escuela vas a poder hacer lo que te guste.

E: ¿Vos pensás lo mismo?

A2: No sé si lo haces, pero tenés más posibilidad. (ASB, 5año)

E: Y eso ¿dónde lo pudieron observar? Tienen por ejemplo amigos que no terminaron...

A1: Mi hermano no terminó y es papá ahora....

A2: Capáz que trabajás, pero no es un trabajo, algo que te gusta hacer [...] Yo no creo que a todo el mundo le guste ser albañil porque es un trabajo cansador, bah, todos son cansadores, pero ese es muy duro. (ASB, $5^{\circ}$ año)

E: ¿Para qué pensás que te sirve venir a la escuela?

A: Para terminar la secundaria y para poder ser alguien en la vida. Si no, no sos nada. Si no tenés la secundaria, querés buscar un trabajo y no conseguís. No sos nada. Tenés que estar trabajando de albañil o cortando pastos por ahí. No sirve de nada eso. (ASB, 5ำaño) 
Los ASM consideraron, a diferencia de los ASB, que sin el título secundario no se puede estudiar ni trabajar. Los trabajos que para los ASB son cercanos y posibles, en caso de no concluir los estudios, se consideran poco convenientes y lejanos como experiencia posible para este otro grupo de alumnos.

E: ¿Para qué piensan que les sirve venir a la escuela?

A3: Tener un trabajo decente.

A1: Ni basurero podés ser si no terminás. (ASM, 4ํaño)

Los ASB, a diferencia de los ASM, hacen alusión a las experiencias de aquellos pares -ya sean ex compañeros, familiares o conocidos del barrio- que no terminaron la escuela. Esas historias son cercanas y no se reconocen como casos aislados. Como se expuso anteriormente, la deserción escolar se da con frecuencia entre los ASB y, según los relatos, está vinculada a la maternidad o paternidad temprana o bien a la necesidad de obtener ingresos a través de actividades que no demandan competencias escolares ni un título.

E: ¿Y los que no terminan la escuela? ¿Qué hacen?

A2: Y... hacen changas o se rebuscan. $\mathrm{O}$ hay algunos que...

A1: No hacen nada.

A2: Claro, no les importa nada.

E: Son los que se dedican a fumar.

A2: Claro. (ASB, $4^{0}$ año)

**

E: ¿Qué diferencia sienten ustedes con los que no han podido estudiar o que dejaron la escuela?

A2: Un futuro.

A1: Y sí, un futuro. Porque hay muchas chicas que dejaron solo porque tuvieron familia. 
E: Sí, eso nos dijeron sus compañeros el otro día, que quedaron embarazadas y después no siguen estudiando.

A1: Sí, dejan todo.

E: ¿Qué hacen esas chicas además de...?

A1: Las que conocí quedaron embarazadas y dejaron...

A2: Terminan laburando en la calle.

A1: Sí.

A2: Porque ninguna se pone a limpiar casas ni nada. Terminan laburando en la calle.

A1: El marido las deja con el hijo y ellas se tienen que rebuscar con cualquier cosa, con lo primero que encuentren.

A1: Buscan lo más fácil que ellas puedan hacer. No terminan la escuela y tienen que buscar lo primero que agarren (ASB, $4^{\circ}$ año). **

$\mathrm{E}: \mathrm{Y}$, ¿otros que no estudien y se dediquen a otra cosa? A trabajar o a otra actividad.

A2: Sí, albañil, lo único. Albañil o limpiando casas.

A1: Las chicas limpiando casas y los hombres son más albañil, así...

A2: En construcción.

A1: No entran con todo lo que necesitan, pero entran. Entran por medio de ayuda de algún amigo, primo, pariente que tienen trabajando.

$\mathrm{E}: \mathrm{Y}$ ellos, ¿terminaron la escuela?

A1: No. Ellos no. (ASB, $4^{\circ}$ ) 
Los ASB, en términos generales, asocian la conclusión de los estudios secundarios a la posibilidad de tener un futuro mejor. Las personas que terminan el secundario tienen mayores y mejores posibilidades laborales, mientras que aquellos pares que no llegan a esa instancia "no son nada". Sin embargo, consideran que la obtención del título no es condición suficiente para poder realizar trabajos "como la gente" y que les permitan tener un "sueldo" y sus "cosas". Un ejemplo de dicho quiebre lo representan aquellos jóvenes egresados consumidores de drogas.

A1: Desde que yo vengo acá he conocido personas que han terminado quinto año y dicen: "Ah, yo voy a estudiar esto", y vos los ves ahora y no son nada. Tienen como 24 años y tienen 4 hijos. Y así están, con tatuajes en las manos, todo. Entonces...

$\mathrm{E}:$ ¿Chicos que terminaron la escuela?

A2: Que a algunos les ha ido bien y a otros no.

$\mathrm{E}: \mathrm{Y}$ a los que les fue bien, ¿Qué hicieron?

A2: Y, tienen un trabajo como la gente. Su sueldo, sus cosas.

E: ¿Qué sería un trabajo como la gente? ¿Cuáles son esos trabajos?

A2: Por ejemplo, uno trabaja en una panadería. El otro está estudiando para policía ahora de grande, pero por eso tienen sus cosas.

E: ¿Y viven acá en el barrio?

A1: Sí.

E: A los chicos que no les fue bien ¿Qué pasó? ¿Qué no hicieron?

A1: Por la droga.

$\mathrm{E}:$ ¿Ya se drogaban terminando el secundario?

A1: Sí, terminando el secundario, sí. Una vez que agarrás la droga no la soltás más. (ASB, 5ำ año) 
En síntesis, los ASB asumen que con el título tienen más posibilidades de realizar trabajos más gratificantes, con un cierto nivel de formalidad y de mayor reconocimiento social. Aquellos jóvenes que por diferentes motivos han abandonado la escuela (maternidad/paternidad temprana, drogadicción, necesidad temprana de obtener ingresos) terminan realizando trabajos sacrificados, informales y poco reconocidos socialmente. Sin embargo, los ASB observan que el título aumenta las posibilidades de éxito, pero no las garantiza, pues algunos de sus pares que han terminado el secundario, pero que consumen drogas, siguen trayectorias similares a las de aquellos que abandonan la escuela en forma temprana.

\section{A MODO DE CONCLUSIÓN}

Este artículo indagó acerca de experiencias educativas exitosas de jóvenes de sectores socioeconómicos bajos que se encuentran en contextos desfavorables para concluir la educación obligatoria. Específicamente, en el modo en el que los jóvenes que no abandonan la escuela articulan diferentes lógicas de acción en relación al barrio, a la familia, al grupo de pares, como así también sus percepciones acerca de los aprendizajes adquiridos y el vínculo entre la secundaria y el mundo universitario y laboral.

La noción de experiencias educativas exitosas en contextos desfavorables se identificó como una clave de lectura capaz de dar cuenta de la complejidad de los procesos educativos estudiados. Cada escuela, en tanto fragmento de un sistema educativo que no tiene referentes comunes, se presenta como escenario de lógicas de acción que posibilitan que las trayectorias educativas reales de los alumnos confluyan con las teóricas (adaptadas al contexto en el que se encuentran). En tanto se asume que existe una acumulación de desventajas que hacen que el tránsito por la escuela no logre superar los desiguales puntos de partida, la selección de dos espacios escolares diferentes se hizo con el objetivo de poder comparar cómo la experiencia educativa se desarrolla de una manera específica en el grupo bajo estudio.

En cuanto a las lógicas de acción reconocidas, en primer lugar, se observó que los ASB asumen que la escuela es un lugar seguro, que los protege de los peligros que perciben en el barrio. En relación a los pares, registran una cercanía con aquellos que continúan estudiando y un distanciamiento y lectura negativa acerca de los que, por diferentes motivos, abandonaron en forma temprana. Por último, la familia tiene un lugar central e interviene en la trayectoria educativa, principalmente a través de un interés manifiesto ( $y$ en acciones asociadas a este) que permite que la continuidad educativa de sus hijos o menores a cargo sea posible.

En relación a las percepciones acerca de los aprendizajes adquiridos, los ASB dijeron que en la escuela incorporan habilidades (comunicativas y de escritura) y conocimientos (nuevas palabras y capacidad de hacer cuentas) que de otra forma no podrían obtener. Por su parte, la universidad aparece como un "destino a seguir", pero en los relatos concretos se observa y evidencia la desarticulación que existe entre ambos 
niveles educativos. Por último, en lo que refiere al trabajo, asumen que la escuela les otorga más posibilidades de tener un trabajo seguro y menos sacrificado que los que realizan sus pares que han abandonado los estudios.

\section{REFERENCIAS}

Anderson, D. (1993). Effects of pregnancy, childbirth, and motherhood on high school dropout. (Institute for Research on Poverty Discussion Paper No. 1027-93). Recuperado de http://www.ssc.wisc.edu/irpweb/publications/dps/pdfs/dp102793.pdf

Aristimuño, A. (2009) El abandono de los estudios del nivel medio en Uruguay: un problema complejo y persistente. Revista Iberoamericana sobre Calidad, Eficacia y Cambio en Educación, 7(4),180198.

Bertaux, D. (2005). Los relatos de vida. Perspectiva etnosociológica. Barcelona, España: Ediciones Bellaterra.

Bourdieu, P., y Passeron, J.C. (2003). Los Herederos. Los estudiantes y la cultura. Buenos Aires, Argentina: Siglo XXI.

Carusso, M., y Dussel, I. (1996). De Sarmiento a los Simpsons: Conceptos para pensar la escuela contemporánea. Buenos Aires, Argentina: Kapelusz.

Cervini, R. (2009). Comparando la inequidad en los logros escolares de la educación primaria y secundaria de Argentina. Un estudio multinivel comparativo. REICE, 7(1), 5-21.

Del Cueto, C. (2007). Los únicos privilegiados. Estrategias educativas de familias residentes en countries y barrios cerrados. Buenos Aires, Argentina: Prometeo.

Dirección Nacional de Información y Evaluación de la Calidad Educativa. (2004). Las dificultades en las trayectorias escolares de los alumnos. Un estudio en escuelas de nuestro país. Buenos Aires, Argentina: Ministerio de Educación, Ciencia y Tecnología-DINIECE.

Dubet, F. (2007). El declive y las mutaciones de la institución. Revista de Antropología Social, 16, 39-66.

Dubet, F., y Martuccelli, D. (1998). En la escuela. Sociología de la experiencia escolar. Buenos Aires, Argentina: Losada.

Elder, G. (1978). Family History and the Life Course. En T. K. Hareven (Ed.), Transitions: the family end the life course in Historical Perspective (pp. 17-57). Nueva York, NY: Academic Press.

Fernández Aguerre, T. (2009). La desafiliación en la educación media en Uruguay. Una aproximación con base en el panel de estudiantes evaluados por PISA 2003. REICE, 7(4), 164-179.

Fernández Aguerre, T. (Coord. y Ed.). (2010). La desafiliación en la Educación Media Superior de Uruguay: conceptos, estudios y políticas. Montevideo, Uruguay: Tabaré Fernández Aguerre Editor.

Hareven, T. K. (2000). Families, History, and Social Change. Life Course and Cross-Cultural Perspectives. Boulder, CO: Westview Press.

Hernández, R., Fernández, C., y Baptista, L. P. (2003). Metodología de la Investigación. México D.F., México: McGraw-Hill Interamericana. 
Lahire, B. (2007). Infancia y adolescencia: de los tiempos de la socialización sometidos a constricciones múltiples. Revista de Antropología social, 16, 21-38.

Lahire, B. (2010). Transmisiones intergeneracionales de la escritura y desempeño escolar. En J. Vaca Uribe (Coord.), Prácticas de lengua escrita: vida, escuela, cultura y sociedad (pp. 13-51). Xalapa, México: Universidad Veracruzana.

Mayer, K. U. (2004). Whose lives? How history, societies, and institutions define and shape life courses. Research in Human Development, 1, 161-187.

Mayer, L. (2009). Escuela, integración y conflicto. Notas para entender las tensiones en el aula. Educación, Lenguaje y Sociedad, 6(6), 85-112.

Maxwell, J. (1996) Qualitative research design. An Interactive Approach. Thousand Oaks, CA: Sage.

Observatorio Argentino de Drogas. (2010). Escolaridad, trabajo y proyecto de vida. La entrada al mundo adulto y los factores de riesgo y protección en el consumo de drogas en adolescentes de 15 a 18 años en AMBA y provincia de Buenos Aires. Recuperado de http://www.observatorio.gov.ar/media/k2/attachments/EscolaridadZTrabajoZyZProyectoZdeZVida .ZAoZ2010.pdf

Pascual, L. (2008) El contexto familiar y su acción mediadora en el desarrollo cognitivo y el rendimiento escolar. Propuesta Educativa, 15(29), 73-82.

Román, M. (2009) Enseñanza media ¿Quiénes y por qué abandonan definitivamente el liceo en Chile? REICE, 7(4), 95 -119.

Salvia, A. (Dir.). (2009). Grandes desigualdades sociales en las oportunidades de socialización más allá de la escuela. Observatorio de la Deuda Social de la Infancia, Boletín № 2. Recuperado de http://bibliotecadigital.uca.edu.ar/repositorio/investigacion/grandes-desigualdades-socialesoportunidades.pdf

Saraví, G.A. (2004). Segregación urbana y espacio público: los jóvenes en enclaves de pobreza estructural. Revista de la CEPAL, 83, 33-48.

Tenti, E. (junio, 2000). Culturas juveniles y cultura escolar. Documento presentado en el Seminario "Escola Jovem: un novo olhar sobre o ensino médio" del Ministerio de Educación, Brasilia, Brasil. Recuperado de http://www.ses.unam.mx/curso2009/materiales/m4/s2/M4 Sesion2 Tenti.pdf

Terigi, F. (2015). Aportes de la investigación sobre políticas educativas y trayectorias escolares en la escuela secundaria. En D. Pinkasz (Comp.), La investigación sobre educación secundaria en la Argentina en la última década (pp. 94-108). Buenos Aires, Argentina: Flacso Argentina.

Tilly, C. (1998). La desigualdad persistente. Buenos Aires, Argentina: Manantial.

Tiramonti, G. (2005) La trama de la desigualdad educativa. Conferencia de Guillermina Tiramonti. Diálogos pedagógicos, 3(5), 94-110. 